\title{
Evidence of a long-term trend in total solar irradiance ${ }^{\star}$
}

\author{
C. Fröhlich
}

\begin{abstract}
Physikalisch-Meteorologisches Observatorium Davos, World Radiation Center, 7260 Davos Dorf, Switzerland e-mail: cfrohlich@pmodwrc.ch
\end{abstract}

Received 13 April 2009 / Accepted 16 June 2009

\begin{abstract}
Aims. During the solar minimum of 2008, the value of total solar irradiance at $1 \mathrm{AU}$ (TSI) was more than $0.2 \mathrm{Wm}^{-2}$ lower than during the last minimum in 1996, indicating for the first time a directly observed long-term change. On the other hand, chromospheric indices and hence solar UV irradiance do not exhibit a similar change.

Methods. Comparison of TSI with other activity parameters indicates that only the open solar magnetic field, $B_{\mathrm{R}}$, observed from satellites at $1 \mathrm{AU}$ show a similar long-term behaviour. The values at the minima correlate well and the linear fit provides a direct physical relationship between TSI and $B_{\mathrm{R}}$ during the minimum times.

Results. This correlation allows an unambiguous reconstruction of TSI back in time, provided the open solar magnetic field can be determined from e.g. geomagnetic indices or cosmogenic radionucleides. Since the solar UV irradiance has no long-term trend, the mechanism for the secular change of TSI must differ from the effect of surface magnetism, as manifested by sunspots, faculae, and network which indeed explain well the intra-cycle variability of both total and spectral irradiance.

Conclusions. The long-term trend of TSI is most probably caused by a global temperature change of the Sun that does not influence the UV irradiance in the same way as the surface magnetic fields.
\end{abstract}

Key words. Sun: activity - sunspots - Sun: UV radiation - Sun: faculae, plages - Sun: magnetic fields - Sun: photosphere

\section{Introduction}

During the 17 th century, unusually cold temperatures were observed in Europe and the solar activity seemed to have almost ceased, as manifested by only a few sunspots during 1645-1680. This period of low activity was called "Maunder Minimum" (MM) by Eddy (1976), who also suggested that the low activity may have been the reason for the low total solar irradiance (TSI). This period has become the most famous test for a possible relation between TSI, solar activity and the Earth's climate. TSI reconstructions back to the 17th century are normally based on two assumptions: (i) the surface manifestations of solar activity such as sunspots, faculae, and network determine the cycle amplitude; (ii) the existence of a long-term, secular variation somehow related to the strength of activity (e.g. Hoyt \& Schatten 1993; Lean et al. 1995; Wang et al. 2005; Krivova et al. 2007; Tapping et al. 2007). Reliable TSI measurements started in 1978 and now cover almost three solar cycles. During the solar minimum of 2008 the value of TSI is more than $0.2 \mathrm{Wm}^{-2}$ lower than it was during the last minimum in 1996, indicating for the first time a directly observed long-term change in TSI. None of the other manifestations of activity, such as sunspot number, microwave flux from the Sun at $10.7 \mathrm{~cm}$ (F10.7), or the chromospheric indices from CaII or MgII and related to them the UV and EUV irradiance showed a decrease of similar magnitude.

In Sect. 2, we discuss the data and estimate the uncertainty in this new result. Section 3 describes the relation to the open field, which is the only activity-related parameter showing such a trend. Section 4 discusses the amplitude variation and the

\footnotetext{
* Appendix is only available in electronic form at http: //www . aanda.org
}

conclusions are presented in Sect. 5. The URLs of the data sets used here are listed in Data Sources at the end of the paper.

\section{Data}

TSI measurements with electrically calibrated radiometers from space started with the launch of NIMBUS 7 in November 1978 and continue today with overlapping measurements from different spacecrafts. From these measurements, three composite time series were constructed, referred to as PMOD (Fröhlich 2006), ACRIM (Willson \& Mordvinov 2003), and IRMB (Dewitte et al. 2004) composites. A major difference between the three composites is the way in which the gap between the end of ACRIM I and the beginning of ACRIM II observations is bridged. Since the first publication of the ACRIM composite by Willson (1997), it has often been asked whether a change in the radiometer HF on NIMBUS 7 occurred during the ACRIM-gap period as first suggested by Lee III et al. (1995) and Chapman et al. (1996). The possibility of this change is still neglected by the ACRIM team and thus their TSI increases by more than $0.5 \mathrm{Wm}^{-2}$ during cycle 22. A detailed reanalysis of the HF long-term behaviour by Fröhlich $(2000,2006)$ and comparison with ERBE data and the Kitt-Peak reconstruction (Wenzler et al. 2006) confirm the need of the correction of HF. The results of another reconstruction (Krivova et al. 2007) were used to "resolve" this issue by Scafetta \& Willson (2009). Since this reconstruction was never designed to reproduce the details of the intra-cycle variability properly, these new arguments are only misleading and do not support the increase in TSI during cycle 22. The consistent corrections of the HF observations over the entire period provide not only an accurate time series over the ACRIM gap, but also 


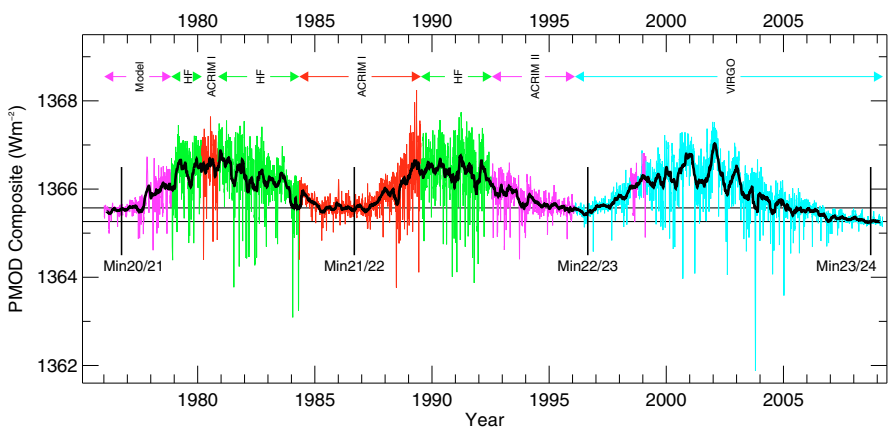

Fig. 1. Daily TSI of the PMOD composite (updated until end of March 2009, Version 41_62_0904) and extrapolated with a proxy model back to 1976. The amplitudes of the three cycles decrease first and then increase again. The two horizontal lines indicate the value of the minima in 1986 and 2008, respectively. Note the low value of the 2008 minimum, which is $0.22 \mathrm{Wm}^{-2}$ lower than the previous one, or $22 \%$ in terms of the cycle amplitude.

a reliable TSI time series during the maximum of cycle 21 (for details see Fröhlich 2003, 2006). Thus, the PMOD composite (version 41_62_0904; the 4 digits in the versions indicate the date of the last update; 41 means V4.1 of the composite and 62 V6.2 of VIRGO) is an accurate TSI record during the last three solar cycles. As shown in Fig. 1, it can also be extended back to the minimum in 1976 with a proxy model from Fröhlich (2009); the validity of this extrapolation is discussed later.

For the following discussion, we need to define the amplitude and point in time of solar minima: "amplitude" is the sine square equivalent, namely twice the mean value of the increase above the solar minimum values averaged over the period between two minima and "time of minimum" is the first appearance of an important sunspot group of the new cycle (at high latitude and of opposite polarity); the last five minima are at: 24 Dec. 1964, 1 Oct. 1976, 7 Sep. 1986, 25 Aug. 1996, and 21 Sep. 2008.

Before discussing the long-term change in TSI we need to demonstrate that the low value is indeed real. The VIRGO data from 18 Jan. 1996 until 31 Mar. 2009 (Fröhlich et al. 1997) used here are from V6.2, which has been updated from V6.1 used earlier by Fröhlich (2008). The difference between these two versions is important and related to the way corrections are applied to PM6V-B, one of the reference radiometer of VIRGO. A description of how VIRGO was analyzed was given in Fröhlich (2003) and in the online Appendix A with special emphasis on the version change from 6.1 to 6.2 . For ACRIM, a combination of measurements from ACRIM II acquired between 4 Oct. 1991 and 13 Sep. 2000 (version of 10/01, Willson 1994) and ACRIM III from 13 Jun. 2000 until 1 Oct. 2008, (version of 11/08, Willson 2001) is used. ACRIM III values are shifted to the level of ACRIM II with the ratio determined over a period of three months before ACRIM III assumed full control on 13-Sep-2000. TIM data (Kopp \& Lawrence 2005) of version 9 are used for the period from 4 Mar. 2003 until 4 Apr. 2009. The three time series are shown in Fig. 2 with their ratios to VIRGO in Fig. 2b. The short to medium term variation of ACRIM is larger than the one of VIRGO and TIM and it shows an oscillation with a period close to one year and an amplitude of up to 300 ppm peak-to-peak. The standard deviation from the linear fit of the ACRIM/VIRGO $(A / V)$ ratio is $\sim 85 \mathrm{ppm}$, whereas that for TIM/VIRGO $(T / V)$ is much lower with $28 \mathrm{ppm}$. This indicates that $A / V$ is dominated by the noise of the ACRIM time series,

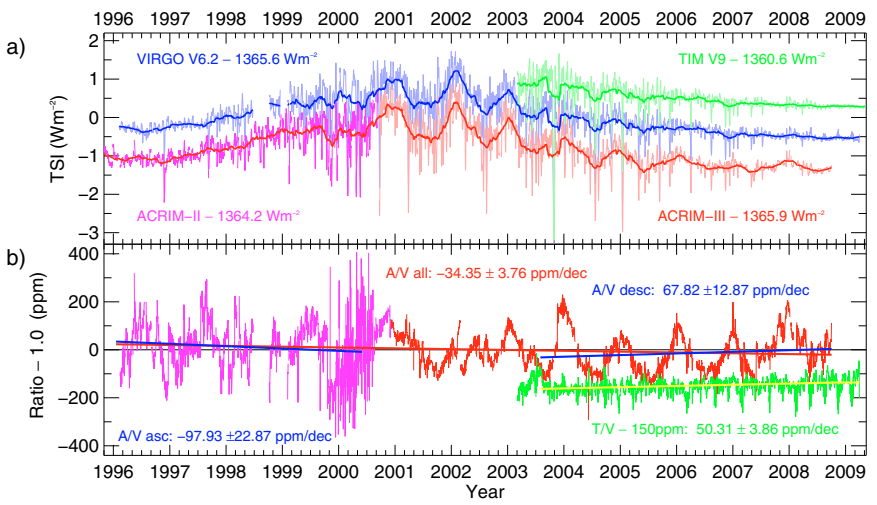

Fig. 2. Comparison of three independent TSI records during solar cycle 23: (a) daily values of TSI from VIRGO (V6.2), ACRIM (combination of ACRIM II and III, version 0811) and TIM (V9) together with a 81-day running mean; and (b) daily ratios of ACRIM and TIM to VIRGO. The slopes are calculated for the full period for overlapping ACRIM and VIRGO data, the ascending and descending part of cycle 23, with the latter starting in August 2003 (first TIM data used here). The uncertainties in the slopes are formal statistical errors.

and $T / V$ most likely provides a good measure of the noise of VIRGO.

The non-zero slope of $A / V$ over the full period may indicate a difference between the two minima. However, this difference could be caused by ACRIM only and the comparison of the slopes from version 6.1 and 6.2 is indeed indicative of the stability of VIRGO. Although the difference may be an upper limit, it can be used as an estimate of the solar-cycle uncertainty in the VIRGO record. This difference can be directly determined from the minimum values of the two versions and amounts to $83.7 \mathrm{ppm}$. The slopes during the ascending and descending phases may indicate that the cycle amplitude of VIRGO is about $50 \mathrm{ppm}$ higher than the one of ACRIM, which is probably related to the incomplete correction of PMO6V-B. This difference does not influence the change between minima. A further contribution comes from the correction over the SOHO vacation which is estimated as $39 \mathrm{ppm}$. For a detailed discussion we refer to Appendix A. Moreover, instrumental noise, determined to be the standard deviation of the $T / V$ fit, must also be included (28.3 ppm, divided by $\sqrt{3 \times 27}$ yielding $3.14 \mathrm{ppm}$ ). The rms sum of these three contributions yields a total uncertainty of the solar-cycle trend of $92 \mathrm{ppm}$. For cycle 22, a value of $70 \mathrm{ppm}$ was estimated (Fröhlich 2004). The minimum value in 1986 is used as reference for the comparison of the relative levels of the other minima and so, the uncertainty is estimated from the $\mathrm{A} / \mathrm{V}$ noise (divided by $\sqrt{3 \times 27}$ ) as $9 \mathrm{ppm}$. The observed change in the VIRGO TSI from 1996 to 2008 of 140 ppm is significant at the $1.5-\sigma$ level. With the minimum in 1986 as reference, the uncertainty of the four minima amounts to $70,9,70$ and $\sqrt{70^{2}+92^{2}}=116 \mathrm{ppm}$ and the corresponding TSI values are $1365.52 \pm 0.10,1365.57 \pm 0.01,1365.45 \pm 0.10$, and $1365.26 \pm 0.16 \mathrm{Wm}^{-2}$, respectively.

\section{Correlation with the open solar magnetic field}

Figure 3 shows the times series of the sunspot number (SSN), the solar radio flux at $10.7 \mathrm{~cm}$ (F10.7), the Ly- $\alpha$ composite (starting with the measurements of AE-E (Woods et al. 2000)), and the chromospheric indices CaII K and MgII. Table 1 summarizes the values of the amplitudes and the relative changes of the minima. None of these time series show changes in the minima and the 
Table 1. Cycle amplitudes in units of the parameter and relative changes of minima in percent of the corrected amplitude during cycle 22 .

\begin{tabular}{lcccc}
\hline \hline Parameter & Cycle 20 & Cycle 21 & Cycle 22 & Cycle 23 \\
\hline \multicolumn{5}{c}{ Corrected cycle amplitude } \\
TSI & $\ldots$ & 0.9324 & 0.8601 & 0.8936 \\
$B_{\mathrm{R}}$ & $\ldots$ & 1.241 & 1.360 & 1.075 \\
SSN & 95.5 & 141.0 & 132.9 & 98.8 \\
F10.7 & 76.9 & 125.6 & 120.7 & 96.2 \\
Ly- $\alpha$ & $\ldots$ & 3.45 & 3.16 & 2.65 \\
CaII K & $\ldots$ & 0.01269 & 0.00987 & 0.00827 \\
MgII & $\ldots$ & 0.0176 & 0.0150 & 0.0138 \\
\hline \multicolumn{5}{c}{ Change of minima over each cycle } \\
TSI & $\ldots$ & 5.6 & -14.2 & $-\mathbf{2 1 . 8}$ \\
$B_{\mathrm{R}}$ & $\ldots$ & 9.8 & -23.7 & $\mathbf{- 3 5 . 3}$ \\
SSN & 0.8 & 0.6 & -3.7 & -4.8 \\
F10.7 & -0.7 & 1.4 & -0.0 & -4.6 \\
Ly- $\alpha$ & $\ldots$ & -1.3 & -2.9 & -1.5 \\
CaII K & $\ldots$ & -1.8 & -2.7 & -2.1 \\
MgII & $\ldots$ & 0.3 & 2.4 & -1.4 \\
\hline
\end{tabular}

${ }^{a}$ Units are: TSI $\left(\mathrm{Wm}^{-2}\right)$, the open field at $1 \mathrm{AU} B_{\mathrm{R}}(\mathrm{nT}), \mathrm{SSN}$ (number), F10.7 ( $\left.\mathrm{sfu} \equiv 10^{-22} \mathrm{Wm}^{-2} \mathrm{~Hz}^{-1}\right), \mathrm{Ly}-\alpha\left(\mathrm{mWm}^{-2}\right)$, CaII K and MgII indices (core-to-wing ratio). The amplitudes are equivalent sine-squared in units of the parameter, and the trend during each cycle is removed.

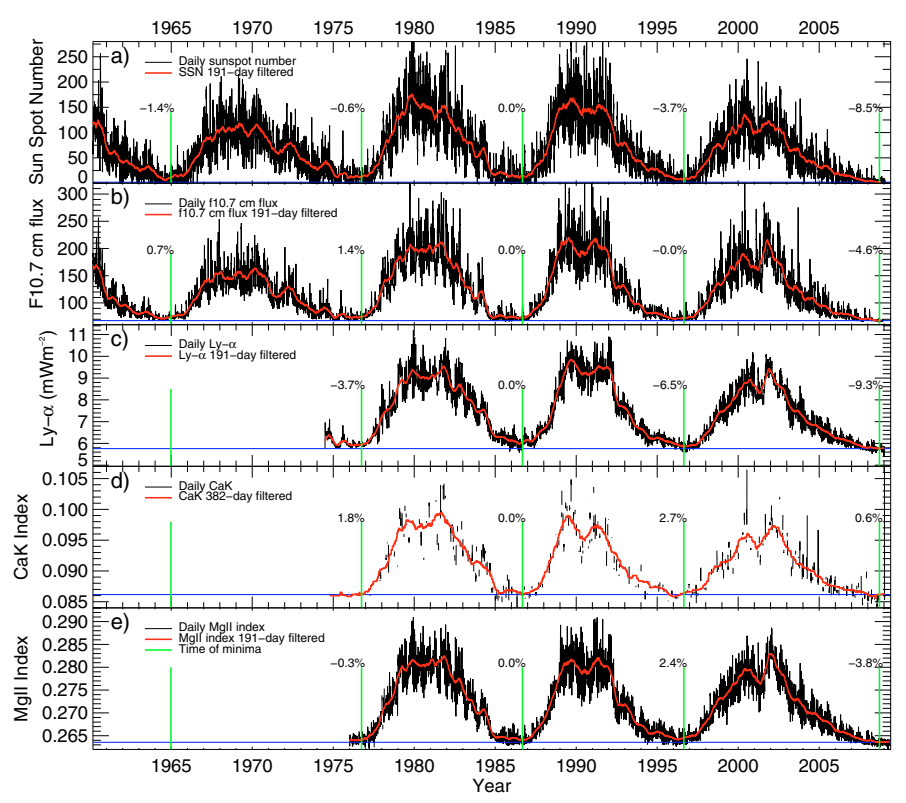

Fig. 3. Daily values of the time series of a) SSN, b) F10.7, c) Ly- $\alpha$ irradiance; d) CaII K; and e) MgII indices. The values indicated at solar minima (above the vertical lines) are the percent changes relative to the amplitude of cycle 22 and normalized to the value between cycles 21 and 22 indicated by the horizontal blue line. In contrast to TSI shown in Fig. 1 and 4 a, none of these records show the large decrease during the present minimum.

variations are at least a factor of 6 smaller. For SSN, this is expected, but the others have no lower limit to their values and thus could show a change. For F10.7, there is also no significant change back to the start of the measurements in 1947 . The CaII K index deduced from the Mt Wilson observations (Foukal et al. 2009b) back to around 1915 shows also no change in the minima to within $\pm 4 \%$. As the chromospheric indices are highly correlated with the UV and EUV irradiance, secular changes of the spectral irradiance below about $300 \mathrm{~nm}$ are hence unlikely. This is confirmed by observations of $\mathrm{Ly}-\alpha$ irradiance during the last
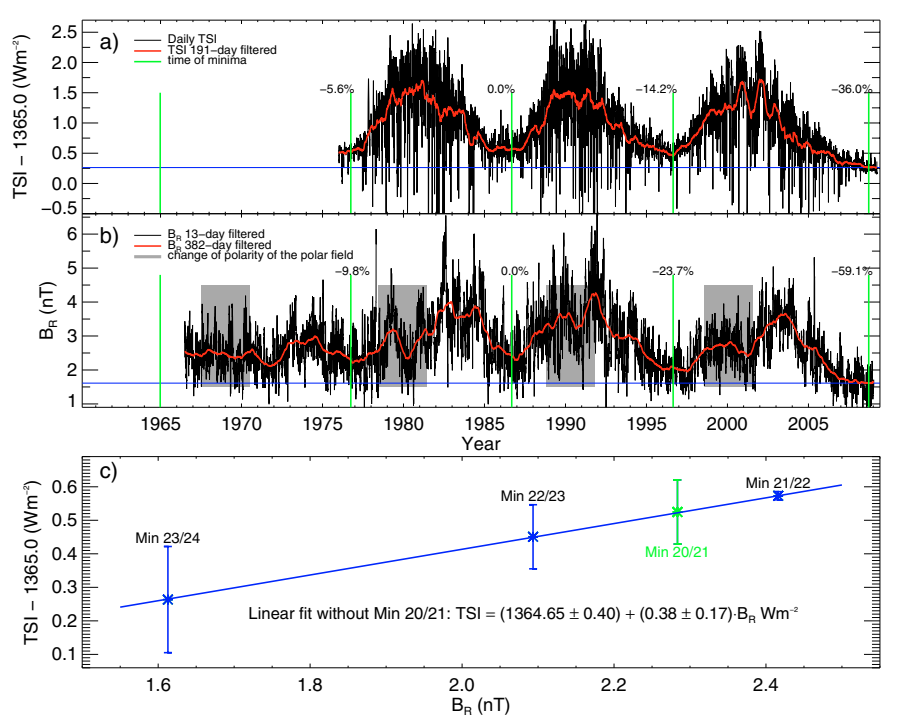

Fig. 4. Comparison of TSI and the open solar magnetic field. a) daily values of TSI (as Fig. 1); and b) those of the open magnetic field $B_{\mathrm{R}}$ at $1 \mathrm{AU}$. The correlation between the minimum values of TSI and those of $B_{\mathrm{R}}$ (blue points and the blue line) is shown on c). The green point is the extrapolated value not used in the regression.

three cycles, and observations of sun-like stars (Giampapa et al. 2006; Hall \& Lockwood 2004; Judge \& Saar 2007). All these observations show that the chromospheric indices and hence the "quiet" magnetic Sun does not change on secular timescales (see also Livingston \& Wallace 2003) and they contradict the normally used assumption that the long-term changes in the solar irradiance are caused by the same mechanism as the intra-cycle variation, namely manifestations of the surface magnetic fields.

The only parameter that does exhibit inter-cycle changes similar to TSI is the open magnetic field, $B_{\mathrm{R}}$, as observed on Earth by satellites since 1963 (Fig. 4b). We determine $B_{\mathrm{R}}$ by taking the absolute value of the daily mean of $B_{\mathrm{X}}$ from the OMNI2 dataset.The intra-cycle variation in $B_{\mathrm{R}}$ is not similar to TSI, the main reason being that $B_{\mathrm{R}}$ changes sign around the maximum of the cycle (during periods of the shaded areas in Fig. 4b), and since the northern and southern hemisphere may change at different times, it is a prolonged period during which the behaviour of the $B_{\mathrm{R}}$ variability is dominated by the reversal. During the minima, $B_{\mathrm{R}}$ is more stable and mainly determined by the polar field, which is representative of the amount of global magnetism remaining from the just passed solar cycle and available for the next one; hence it is a measure of prevailing strength of the activity. The minimum values of TSI and $B_{\mathrm{R}}$ (also listed in Table 1) do correlate quite well as shown in Fig. $4 \mathrm{c}$, and from a linear fit the TSI- $B_{\mathrm{R}}$ sensitivity can be deduced as $S\left(B_{\mathrm{R}}\right)=(1364.64 \pm 0.40)+(0.38 \pm 0.17) B_{\mathrm{R}}$ in $\mathrm{Wm}^{-2}$. This regression does not include the value of the proxy-based minimum in 1976, but its position close to the linear fit confirms the validity of this extrapolation over the 2.5 years with the proxy model. With the TSI- $B_{\mathrm{R}}$ calibration and a reconstruction of the open magnetic field from geomagnetic indices (Lockwood et al. 1999; Rouillard et al. 2007; Svalgaard \& Cliver 2007), TSI can be determined from $B_{\mathrm{R}} \approx 1.1 \mathrm{nT}$ at the minimum of $1912 / 13$ to be $1365.06 \mathrm{Wm}^{-2}$, which is $0.51 \mathrm{Wm}^{-2}$ lower than during the minimum of 1986 . During the MM, $B_{\mathrm{R}} \approx 0.7 \mathrm{nT}$ (e.g. Wang \& Sheeley 2003; Steinhilber et al. 2009) and TSI was $1364.91 \mathrm{Wm}^{-2}$ or $0.66 \mathrm{Wm}^{-2}$ below the minimum of 1986 . We note that our result for the MM is similar to the values of 
other TSI reconstructions (e.g. Wang et al. 2005; Krivova et al. 2007), although the derivations are completely different. Both Wang et al. (2005) and Krivova et al. (2007) use either the total photospheric magnetic field or the sum of the open field and the fields from the ephemeral regions for the secular change and determine it in the same way as for the intra-cycle variability. This implicitly assumes that the UV irradiance also has a trend proportional to the one of TSI, which is incompatible with the observations during the past three cycles.

\section{Cycle amplitude}

The intra-cycle variability in both the UV irradiance and TSI are explained well by manifestations of the surface magnetic fields, sunspots, faculae, and the network. These models (e.g. Wenzler et al. 2006; Fröhlich 2009; Unruh et al. 2008) are based on contrast functions, area, and center-to-limb variations in the effect of the different magnetic elements involved and explain more than $80 \%$ of the variance on time scales from days to 11 years. During the last solar cycle, it was realized that a linear trend must be added to reach this level of correlation (e.g. Fröhlich 2009). Comparison of the TSI amplitudes with those of other parameters listed in Table 1 during the past three cycles shows that TSI is significantly higher in cycle 23 . This is due to extended periods when TSI is increased by large faculae in activeregions without or with only small sunspots. A similar case may have been observed during cycle 20 when the facular area was also high (Brown \& Evans 1980; Foukal 1993) relative to SSN. Cycle amplitude reconstruction into the past needs to be investigated in more detail and the behaviour during cycle 23 is an excellent case for testing new approaches. If one simply uses the correlation between TSI and e.g., SSN to determine the TSI amplitude, the result is within $\pm 18 \%$ for the three observed cycles. So, such a correlation can provide a TSI amplitude within only about $\pm 20 \%$, which may be acceptable in some cases.

\section{Conclusions}

The difference between the long-term behaviour of TSI and the UV spectral irradiance may be caused by a global temperature change, as already suggested by Tapping et al. (2007). From the sensitivity of TSI to changes in temperature $(0.07 \% / \mathrm{K})$ a change of $\approx 0.2 \mathrm{~K}$ during cycle 23 has been deduced, and at the wavelength of Ly- $\alpha$ with about $0.4 \% / \mathrm{K}$, we measure a $1.1 \%$ change, which is negligibly small compared to the cycle variation of more than $60 \%$. Although the difference becomes smaller at longer wavelengths, at $300 \mathrm{~nm}$ the intra-cycle variation is still 4-5 times larger than the temperature change would suggest. Another explanation may be the influence of surface magnetic fields such as polar faculae, which are important during solar minimum with at present the lowest number ever observed (Sheeley 2008). However, estimates of the influence of polar faculae indicate that their influence on TSI is indeed too small (Foukal et al. 2009a).

We conclude that the long-term change in TSI is caused by a global temperature change of about $0.2 \mathrm{~K}$ during cycle 23 . The observed minima of TSI are well correlated with those of $B_{\mathrm{R}}$, which allows the determination of TSI at a cycle minimum for any time with available $B_{\mathrm{R}}$. Furthermore, the UV and EUV irradiances do not show any long-term trend, which has important consequences for our understanding of the solar variability and its influence on global climate change.

Data Sources: PMOD composite: http://www.pmodwrc.ch/pmod.php? topic=tsi/composite/SolarConstant; ACRIM composite: http://www . acrim . com/RESULTS/Composite/composite_acrim_hdr_1108.txt; IRMB composite:
http://remotesensing.oma.be/TSI/SARR.diarad; daily VIRGO TSI: http:// www.pmodwrc.ch/pmod.php?topic=tsi/virgo/proj_space_virgo; daily SSN: ftp://ftp.ngdc.noaa.gov/STP/SOLAR_DATA/SUNSPOT_NUMBERS; daily F10.7: ftp://ftp.ngdc.noaa.gov/STP/SOLAR_DATA/SOLAR_RADI0/FLUX; daily Ly- $\alpha$ : ftp://laspftp.colorado.edu/pub/SEE_Data/composite_lya/composite_lya. dat; CaII K fulldisk data: ftp://diglib.nso.edu/.data11/cycle_spectra/ reduced_data/fd.log2; daily $\mathrm{MgII}$ index originally from ftp://ftp.ngdc. noaa.gov/STP/SOLAR_DATA/SOLAR_UV/NOAAMgII.dat and updated since 2003 from http://lasp.colorado.edu/sorce/ssi_data/mgii/txt; $B_{\mathrm{R}}$ daily averages from OMNI2 database: ftp://nssdcftp.gsfc.nasa.gov/spacecraft_data/ omni.

Acknowledgements. I thank J. Beer, P. Foukal, H. Spruit and F. Steinhilber for many helpful discussions about the data and their interpretation and especially G. Kopp for his contribution to the estimation of uncertainties, which clarified this issue substantially. Thanks are extended to the VIRGO team for its continuous support of the experiment and to the SOHO Operations which made this cycle-long record possible. SoHO is a cooperative mission of ESA and NASA. Last, but not least the long-term support by the Swiss National Science Foundation of this research at PMOD/WRC is gratefully acknowledged.

\section{References}

Brown, G. M., \& Evans, D. R. 1980, Sol. Phys., 66, 233

Chapman, G. A., Cookson, A. M., \& Dobias, J. J. 1996, J. Geophys. Res., 101, 13541

Dewitte, S., Crommelynck, D., Mekaoui, S., \& Joukoff, A. 2004, Sol. Phys., 224, 209

Eddy, J. A. 1976, Science, 192, 1189

Foukal, P. 1993, Sol. Phys., 148, 219

Foukal, P., Bernasconi, P., \& Fröhlich, C. 2009a, SPD Meeting, 2009, Boulder C0. USA

Foukal, P., Bertello, L., Livingston, W., et al. 2009b, Sol. Phys., 255, 229

Fröhlich, C. 2000, Space Sci. Rev., 94, 15

Fröhlich, C. 2003, Metrologia, 40, 60

Fröhlich, C. 2004, in Geophysical Monograph 141: Solar Variability and its Effect on Climate (American Geophysical Union, Washington DC, USA), 97 Fröhlich, C. 2006, Space Sci. Rev., 125, 53

Fröhlich, C. 2008, AGU Fall Meeting Abstracts, abstract: SH21C-05

Fröhlich, C. 2009, in Climate and Weather of the Sun-Earth System(CAWSES): Selected Papers from the 2007 Kyoto Symposium, ed. T. Tsuda, R. Fujii, K. Shibata, \& M. A. Geller (Terra Publishing, Setagaya-ku, Tokyo, Japan), 217 available at http://www.terrapub.co.jp/onlineproceedings/ ste/CAWSES2007/index.html

Fröhlich, C., Crommelynck, D., Wehrli, C., et al. 1997, Sol. Phys., 175, 267 Giampapa, M. S., Hall, J. C., Radick, R. R., \& Baliunas, S. L. 2006, ApJ, 651, 444

Hall, J. C., \& Lockwood, G. W. 2004, ApJ, 614, 942

Hoyt, D. V., \& Schatten, K. H. 1993, J. Geophys. Res., 98, 18895 Judge, P. G., \& Saar, S. H. 2007, ApJ, 663, 643

Kopp, G., \& Lawrence, G. 2005, Sol. Phys., 230, 91

Krivova, N. A., Balmaceda, L., \& Solanki, S. K. 2007, A\&A, 467, 335

Lean, J., Beer, J., \& Bradley, R. 1995, Geophys. Res. Lett., 22, 3195

Lee III, R. B., Gibson, M. A., Wilson, R. S., \& Thomas, S. 1995, J. Geophys. Res., 100, 1667

Livingston, W., \& Wallace, L. 2003, Sol. Phys., 212, 227

Lockwood, M., Stamper, R., \& Wild, M. 1999, Nature, 399, 437

Rouillard, A. P., Lockwood, M., \& Finch, I. 2007, J. Geophys. Res., 112:A05103 Scafetta, N., \& Willson, R. C. 2009, Geophys. Res. Lett., 36:L05701

Sheeley, Jr., N. R. 2008, ApJ, 680, 1553

Steinhilber, F., Beer, J., \& Fröhlich, C. 2009, Geophys. Res. Lett., submitted Svalgaard, L., \& Cliver, E. W. 2007, J. Geophys. Res., 112:A10111

Tapping, K. F., Boteler, D., Charbonneau, P., et al. 2007, Sol. Phys., 246, 309 Unruh, Y., Krivova, N., Solanki, S., Harder, J., \& Kopp, G. 2008, A\&A, 486, 311 Wang, Y.-M., Lean, J. L., \& Sheeley, N. R. 2005, ApJ, 625, 522

Wang, Y.-M., \& Sheeley, N. R. 2003, ApJ, 591, 1248

Wenzler, T., Solanki, S. K., Krivova, N. A., \& Fröhlich, C. 2006, A\&A, 460, 583

Willson, R. C. 1994, in The Sun as a Variable Star, Solar and Stellar Irradiance

Variations, ed. J. Pap, C. Fröhlich, H. S. Hudson, \& S. Solanki (Cambridge

UK: Cambridge University Press), 54

Willson, R. C. 1997, Science, 277, 1963, see also comment by R. Kerr on page 1923 of the same issue of Science

Willson, R. C. 2001, The Earth Observer, 13, 14

Willson, R. C., \& Mordvinov, A. V. 2003, Geophys. Res. Lett., 30, 1199

Woods, T. N., Tobiska, W. K., Rottman, G. J., \& Worden, J. R. 2000,

J. Geophys. Res., 105, 27195 


\section{Appendix A: VIRGO radiometry: Comments on the difference between versions 6.1 and 6.2 and related issues of the analysis.}

ACRIM, VIRGO, and TIM experiments all have at least one backup or reference radiometer per type which are less exposed and used as degradation reference for the operational one. The starting point of the evaluation are level 1 data, which are corrected for all known effects: e.g. temperature, electrical calibration, and distance (an example are the level 1 data in Fig. 1 of the VIRGO URL ${ }^{1}$ ). The first step to level 2 is to adjust the operational to the backup, which is straightforward for DIARAD (left channel (L) is the operational, and the right channel (R) is the backup) by simply using the ratio $\mathrm{L} / \mathrm{R}$ as measured and some adequate interpolation between the values of $\mathrm{R}$ once a month. The values in this way corrected are now called level 1.8. For PMO6V, this is more complicated because the backup was used at the beginning quite often and therefore also shows an early increase, which needs to be corrected before it can be used to correct PMO6V-A, the operational radiometer (see also the VIRGO URL and Fröhlich 2003, 2006). So, we start with determining the early increase correction for PMO6V-A by comparing it with DIARAD, and the coefficients of the hyperbolic functions describing this early increase. All this is achieved in exposure time and the corresponding UV-dose at each point in time. With the data available at this stage, it is difficult to determine the corresponding coefficients for PMO6V-B mainly because its exposure was important and varied during the first two years of operation. The same functions as determined for PMO6V-A were therefore used, but with the corresponding exposure time and dose of PMO6V-B. This is an approximation, and although further analysis showed that it is not really adequate, it has not been changed. With the corrected PMO6V-B, we can determine the level 1.8 for PMO6V and we have two time series that can be compared. From this comparison, it became obvious that DIARAD has some non-exposure dependent change, which obviously cannot be corrected internally, but needs an independent time series, which is PMO6V. It was already stated in the original proposal that two different types of radiometer are incorporated to enable a clearer understanding of the behaviour in space, and indeed we have detected the unexpected non-exposure dependent change in DIARAD. An exponential function with a step over the unknown change during SOHO vacations, explains the difference very well; the presently used fit was finalized for V6.0 in November 2003 and the coefficients have not been changed since. Obviously it could also be that PMO6V is responsible for this difference, but comparison with e.g. ERBE confirms that it is caused by DIARAD (see e.g. Fig. 3 at the PMOD composite $U_{R L}{ }^{2}$ ). There are also changes in DIARAD after switch-off and -on again, for which we have no explanation but need correction. We do not know the cause of these non-exposure changes, although the HF radiometer on NIMBUS 7 exhibits a similar behaviour (Fröhlich 2006).

Until now no external results have been used to evaluate the VIRGO radiometers. Only changes over the period during which SoHO was lost (25 June to 8 Oct. 1998), called SOHO vacations, need external help, since we can only determine the difference between the corrected DIARAD and PMO6V, but not its absolute value. To determine this correction, we use ACRIM-II for comparison during 122 days before the SoHO vacations and

\footnotetext{
1 http://www $\cdot$ pmodwrc.ch/pmod.php?topic=tsi/

virgo/proj_space_virgo

2 http://www.pmodwrc.ch/pmod.php?topic=tsi/composite/SolarConstant
}

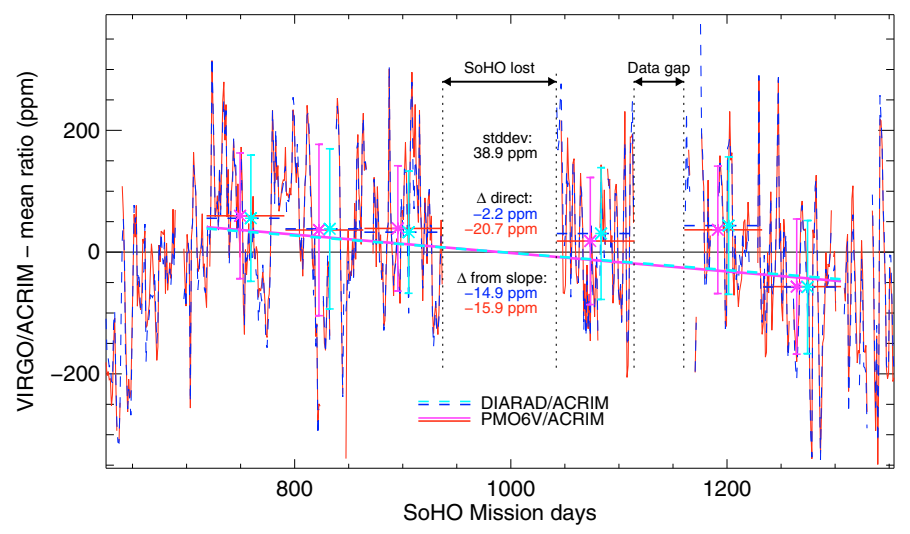

Fig. A.1. Daily ratios of the VIRGO radiometers to ACRIM-II during two years centered on the SOHO vacations. The asterisk with the error bars indicate the mean and standard deviation for each comparison period of 72 days for both VIRGO radiometers. The lines are linear fits to the ratios of DIARAD and PMO6V over the time of the six comparison periods. The results as difference from the adjacent means and the ones determined from the slope are listed in the SoHO gap. Also listed is the standard deviation of the six 72-day means which is used as an estimate for the uncertainty of the SoHO-vacation bridging.

131 days after the data gap, as a compromise between having enough data points and avoiding sudden changes in the ACRIM record. The normal operation immediately after the recovery lasted only 72 days, when the spacecraft was placed in spinning mode. This still provided accurate solar pointing, but no science data transmission until 3 Feb. 1999. Transmission of science data was not possible in this mode of operation because only the low gain antenna, but not the high gain antenna could be used with a very limited data rate. Although we have no data, the instruments were operated normally, which means that this period does not need to be bridged with ACRIM data. In order to have an independent assessment of the uncertainty we use six periods of 72 days for the comparison, three before the summer vacations and one just after, and two after the data gap as shown in Fig. A.1. The results are listed on the plot in the SoHO gap period. From the linear fit we derive a change of about $15 \mathrm{ppm}$ which may be used as a first estimate of the uncertainty. This can be compared to the differences between the 72-day mean just before and after the gap, which show for PMO6V a change of similar magnitude, but for DIARAD a much smaller one. DIARAD has this strange behaviour after a switch-off and -on, and since this interruption is much longer than the three 1-2 days interruptions used to develop the correction (see e.g. Fröhlich 2003), the effect seems to be underestimated, and thus the applied correction is inadequate. Still another way to estimate the uncertainty is to use the standard deviation of the six averages before and after of $38.9 \mathrm{ppm}$. This rather high value is mainly due to the last point, which lies outside the distribution represented by the five remaining points, covering the period of about 280 days before and after the SoHO vacations. Although, the standard deviation of the five points is around $15 \mathrm{ppm}$, we use the larger value of $38.9 \mathrm{ppm}$ for the uncertainty of the change over the SoHO vacation, which is an upper limit.

Shortly after introducing V6.0, it was realized that a slight trend (presently $0.025 \mathrm{ppm} /$ day) in the ratio of the corrected (level 1.8) PMO6V and DIARAD values after the SOHO gap has to be added in the evaluation. At that time, it was attributed to DIARAD because the determination of the exponential function may have needed some adaptation to the data since the original determination. Independently, I started in early 


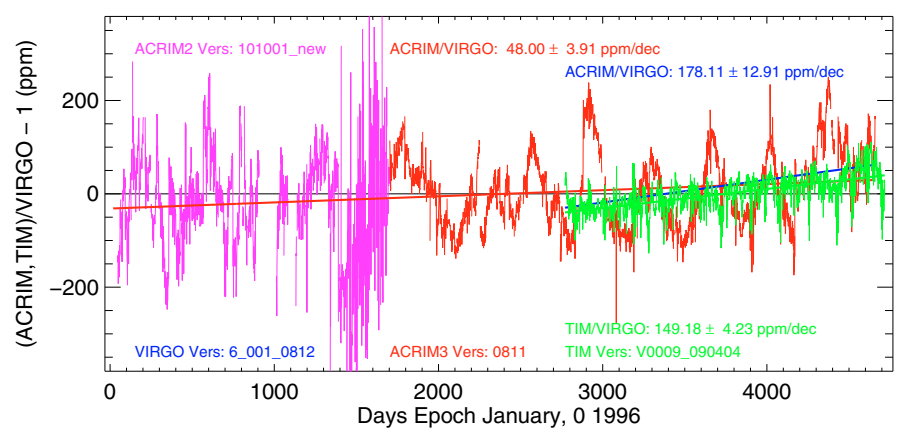

Fig. A.2. Daily ratios of ACRIM (of 0811) and TIM (V9 of 0904) to VIRGO (V6.1 of 0812). The uncertainties of the slopes are formal statistical errors. The difference between ACRIM and VIRGO from minimum to minimum of cycle 23 (12.1 years apart) can be obtained by multiplying the slope given in ppm/decade by 1.21 and amounts to $A / V-1=-39.8 \mathrm{ppm}$.

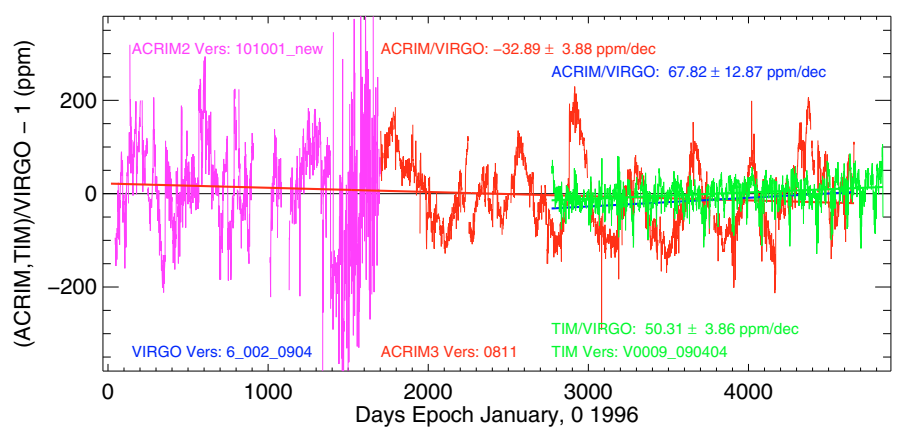

Fig. A.3. Daily ratios of ACRIM (of 0811) and TIM (V9 of 0904) to VIRGO (V6.2 of 0904). The uncertainties of the slopes are formal statistical errors. The difference between ACRIM and VIRGO from minimum to minimum of cycle 23 (12.1 years apart) can be obtained by multiplying the slope given in ppm/decade by 1.21 and amounts to $A / V-1=58.1 \mathrm{ppm}$.

2006 a reanalysis of the whole PMO6V radiometry, which was never completed, but showed that using the early increase coefficients of PMO6V-A for PMO6V-B made its correction too important, especially also since the cycle amplitude and the reanalysis decreased the trend between VIRGO and TIM by about $60 \mathrm{ppm} / \mathrm{dec}$. Although the linear trend is only an approximation to the problem of PMO6V-B, it seems quite reasonable to apply the trend to PMO6V and not to DIARAD.
This attribution of the trend represents the change from V6.1 to V6.2, which was applied following the discussions after the presentation of preliminary results at the AGU Fall Meeting 2008 (Fröhlich 2008) and its influence is shown in Figs. A.2 and A.3. The 81-day average centered around the minimum value in 2008 amounts to 1364.899 and 1364.014 for versions 6.1 and 6.2 respectively, with a difference of $83.7 \mathrm{ppm}$, which is close to the difference determined from the A/V slopes of Figs. A.2 and A.3. The directly determined value is, however, more relevant to the uncertainty in the stability of the VIRGO radiometry, if the version change can indeed be regarded as representative of this uncertainty.

The results of the correlation of TSI with $B_{\mathrm{R}}$ do not change significantly from 6.1 to 6.2 and the results are well within the corresponding uncertainties. Thus, the version change does not influence this correlation and the final result with all points lying on or very close to the fitted line may indeed indicate that the estimated uncertainties may well be upper limits.

There is still another issue that has added to the confusion about the independency of VIRGO TSI, namely the use of ACRIM and TIM in the analysis of the difference between corrected level 1.8 data of PMO6V and DIARAD to provide a reasonable average for the final VIRGO data product. The absolute difference between the two VIRGO radiometers is about $700 \mathrm{ppm}$, but varies with time within about $\pm 80 \mathrm{ppm}$. The question is, whether a simple average is adequate or whether we can use another TSI record as a reference to decide which radiometer may be responsible for a given difference. As described in Fröhlich (2003), we compare the filtered differences (filter with $3 \mathrm{db}$ points at periods of 130 and 460 days) of PMO6V and DIARAD to the reference, which is now a combination of ACRIM II, ACRIM III and TIM data, the latter since March 2003. From the daily difference $\Delta_{\mathrm{P}, \mathrm{D}}=S_{\mathrm{P}, \mathrm{D}}-S_{\text {ref }}$ and a threshold related to the standard deviation of the differences $\delta=4 \sigma$, a distribution function $0 \leq \alpha \leq 1$ is defined as $\alpha=1$ for $\left|\Delta_{\mathrm{P}}\right|>\left|\Delta_{\mathrm{D}}\right|+\delta$ or $\alpha=0$ for $\left|\Delta_{\mathrm{D}}\right|>\left|\Delta_{\mathrm{P}}\right|+\delta$ or as $\alpha=0.5$, if none of the conditions apply. The time series $\alpha$ is then smoothed with a 25-day boxcar and applied to the residual $r$ with $\alpha r$ to PMO6V and with $(1-\alpha) r$ to DIARAD. The final VIRGO TSI is the average of the two $\alpha$-corrected time-series. This assignment of the correction influences the final VIRGO TSI at most on time scales of 130-460 days, but does not change the trend. Although this method reduces the noise of comparisons with other TSI, it may be better to remove it from the analysis and determine VIRGO TSI by means, which would definitively avoid confusion. 